\title{
Sistema cerebral del placer y de la drogodependencia
}

\author{
Rafael A. Ulloque
}

\begin{abstract}
Resumen
Hace cuatro décadas se describió un circuito neuronal que sería el substrato neuroanatómico y neurobioquímico del placer, la recompensa y la drogodependencia. Algunos animales de experimentación son capaces de autoestimularse eléctricamente o de autoadministrarse sustancias de abuso en las áreas de este sistema de una manera compulsiva, produciéndose manifestaciones que se interpretan como conducta placentera. En esta situación, los animales son capaces de abandonar totalmente otras actividades placenteras como la alimentación y el sexo.

El núcleo accumbens, el hipocampo, la corteza prefrontal y la amígdala son los núcleos o áreas cerebrales más importantes de este circuito; el núcleo accumbens (y su fisiología) es el centro crítico de la iniciación y del mantenimiento del refuerzo de la conducta y del abuso de drogas. Allí llegan aferencias de estructuras corticales como la corteza prefrontal y el hipocampo, y de otras como la amígdala y el área ventral del tegmento. El núcleo accumbens proyecta al pálido ventral y a los núcleos motores mesencefálicos desde los cuales salen eferencias a la médula espinal. Esta sería la vía de difusión de estímulos nacidos en el núcleo accumbens, los cuales están involucrados en la actividad sicomotriz. Se enfatizará especialmente la neurotransmisión del núcleo accumbens y del hipocampo.
\end{abstract}

Se piensa que la dopamina y algunos neurotransmisores aminoacidérgicos son importantes en la regulación sináptica de este sistema. Existe consenso sobre el papel relevante de la dopamina en este circuito de recompensa, neurotrasmisor que está muy involucrado en los condicionamientos y en la drogodependencia. Sin embargo, no debe dejarse de lado la función de los otros neurotransmisores.

Esta revisión destaca principalmente el papel que juegan el GABA, el glutamato y el aspartato y, muy recientemente, la participación del oxido nítrico.

Palabras clave: sistema de recompensa, drogodependencia, neurotransmisores, sustancias de abuso.

\section{Reward system and drug dependence}

\section{Abstract}

A neural circuitry which could be the anatomical and neurochemical substrate for pleasure, reward and drug dependence was described four decades ago. Some lab animals are able of compulsive electrical self-stimulation or drug self-administration affecting the nucleus of this circuit, and thus showing a behavior interpreted as pleasure. If per-

Sección de Farmacología, Departamento Básicas II, Facultad de Medicina, Universidad de Cartagena, Cartagena, Colombia. 
mitted, they would permanently seek for this behavior, abandoning feeding or sex.

The nucleus accumbens, the hippocampus, the prefrontal cortex, and the amygdala are the relevant areas of this system. The nucleus accumbens has been suggested as a critical neuronal part for drug reinforcement and drug dependence. Its afferents, involved in the regulation of drug reinforcement, arise from cortical structures of which the prefrontal cortex, the hippocampus, and the amygdaloid complex provide the densest input. The efferent projections from the nucleus accumbens involved in these behavioral events are the ventral pallidum, and the mesencefalic motors (nuclei from which the efferents go to the spinal cord). This efferent pathway helps control motor behaviour.

Dopamine has been pointed out as the principal triggering neurotransmitter to initiate and also maintain reward and drug dependence in these pathways. However, support for the role of GABA, glutamate, aspartate and, very recently, nitric oxide in this pleasure and reward system is reviewed here.

Key words: reward system, drug dependence, neurotransmitters, substances of abuse.

Hace más de cuatro décadas se acepta la hipótesis de la existencia de un sistema neuronal que sería el substrato anatómico y neurobioquímico del placer, el cual puede ser estimulado también por las drogas y los fármacos de abuso. Esta idea sostiene que, en los mecanismos y complicaciones sicológicas y de la conaucta por el abuso de estas sustancias (drogodependencia), este sistema tiene más importancia que los factores sicológicos o sociales. Este circuito neuronal fue descrito por Olds y Milner en 1954 y se concibió después de ensayos de laboratorio, cuando al implantar electrodos en ciertas áreas del sistema nervioso central (SNC) y administrar microestímulos eléctricos, el animal mostraba actitud placentera. Además, cuando los animales de laboratorio tienen la alternativa de autoestimularse en un circuito electrónico cerrado o de autoadministrarse en estas áreas sustancias que producen dependencia, eligen una conducta repetitiva presionando una palanca para recibir un estímulo eléctrico o una dosis de droga, lo cual produce unas manifestaciones que se interpretan como placer.

A este modelo se le conoce como autoestimulación y establece un patrón de trabajo-estímulo; con esta técnica se ha estudiado la acción y los efectos de la dependencia y de la adicción a drogas como los los opioides, el alcohol, las benzodiacepinas, los barbitúricos, las anfetaminas, la cocaína y otras (1).

Hace más de tres décadas y media, se propuso que un sistema monoaminérgico sería la base neurobioquímica de este circuito del placer. A principios de los 60 se comenzaron a publicar artículos que sostenían la idea de que ciertamente la actividad monoaminérgica juega un papel importante en el refuerzo del abuso de algunas drogas y de algunos fármacos. Básicamente, se trata de un sistema que conecta la parte ventral del cerebro medio con la parte ventral y anterior del encéfalo.Entre las conexiones más conocidas están las vías mesolímbica y las mesocorticales que son principalmente dopaminérgicas (2). Existen, además, conexiones claves como las que van de la corteza olfatoria, el septum, el hipocampo y la amígdala al núcleo accumbens, el hipotálamo y la región ventral tegmentaria.

Una de las estructuras más citadas y estudiadas de este sistema de recompensa es el núcleo accumbens. Se piensa que esta estructura juega un papel clave en las acciones y efectos agudos y crónicos de las drogas de abuso. Se considera que la dopamina y otras monoaminas, neurotransmisores aminoácidos y neuropéptidos, tienen gran actuación en las funciones de este centro de refuerzo (3). 


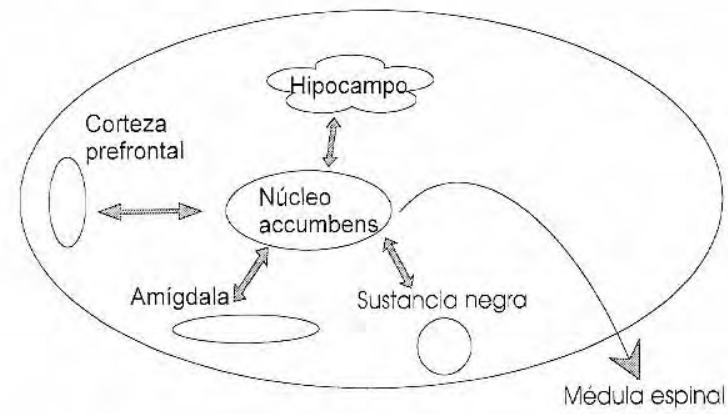

Figura 1. Circuito de recompensa: la corteza prefrontal, el hipocampo, la amígdala, la sustancia negra, interactúan con el núcleo accumbens, el cual envía estímulos a la médula espinal. Este circuito es el responsable en gran parte de las conductas placenteras y de recompensa. Aquí estaría el substrato anatómico, neurobioquímico y neurofisiológico de la drogodependencia.

Además, existen estructuras como el hipocampo, la corteza prefrontal, la corteza entorrinal, la amígdala cerebral y los núcleos motores mesencefálicos, que también son importantes en el refuerzo de conductas y alteraciones neurobioquímicas por drogas de abuso que producen dependencia. En estas áreas cerebrales, tienen importancia los neurotransmisores GABA, el glutamato y el aspartato $(4,5)$ (figura 1).

\section{Núcleo accumbens}

El núcleo accumbens es una estructura telencefálica ubicada en las zonas basales del encéfalo anterior. Se describió en las primeras décadas de este siglo, precisamente durante el auge de las técnicas de marcación e identificación neuronal y de sus proyecciones.

Se considera una extensión ventro-medial del núcleo caudado, por lo cual algunos lo llaman el núcleo estriado ventral. Heimer y Wilson fueron los primeros en llamarlo con este nombre $(6,7)$. Aunque desde el punto de vista anatómico se le considera como parte del núcleo estriado, los estudios de conducta sugieren que está integrado a las funciones del sistema límbico (con el cual presenta muchas interconexiones). Se piensa que tiene funciones relacionadas con la iniciación y regulación de la actividad motora espontánea $y$ en funciones relacionadas con las emociones y la conducta. Se tiene evidencia de su importante papel en los mecanismos neurobioquímicos y neurofisiológicos de la drogodependencia. Se ha dado especial énfasis al papel crítico del núcleo accumbens tanto en los efectos agudos como en los crónicos por cocaína, anfetaminas, derivados opioides y otras drogas (8).

Se considera que los neurotransmisores utilizados por las proyecciones de origen cortical y por los núcleos subcorticales al núcleo accumbens son el glutamato y el aspartato. En el caso de las proyecciones desde el hipocampo, éstas emergen de las células piramidales de las regiones CA1, CA3 y del subiculum, las cuales también utilizan el glutamato y el aspartato como neurotransmisores. Así mismo, llegan al núcleo accumbens aferentes de otras estructuras del sistema límbico como son la corteza prefrontal, la amígdala, el hipocampo, la corteza entorrinal y el girus cingulado (9).

Las proyecciones eferentes del núcleo accumbens terminan en el diencéfalo, la corteza, el tallo cerebral y otras estructuras como el cíngulo, el hipotálamo, el globo pálido, el tálamo y la sustancia negra. Estas utilizan el glutamato y el aspartato como neurotransmisores excitadores y al GABA, regulador del incremento de la actividad sicomotora por la activación del núcleo accumbens,como neurotransmisor inhibidor. Son bien conocidas las abundantes proyecciones dopaminérgicas que llegan a este núcleo, provenientes del área ventral del tegmento. Esta estructura de nivel mesencefálico está muy próxima a la sustancia negra y, como ésta, posee una gran cantidad de neuronas dopaminérgicas que Dahlstrom y Fuke llamaron células $\mathrm{A} 10$.

\section{Conexiones del núcleo accumbens}

\section{Aferencias}

El núcleo accumbens recibe aferencias especialmente de la zona ventral del tegmento, donde existen las neuronas dopaminérgicas (células A8-A10) (10). También recibe proyecciones de alo y perialocorteza de estructuras como el hipocampo, la corteza entorrinal y olfatoria y del complejo amigdalino. Estas proyecciones generalmente son cruzadas y la parte contralateral es de menor abundancia. La distribución 
de las proyecciones aferentes y sus sinapsis no es homogénea. La porción anteromedial del núcleo accumbens recibe axones del subiculum (parte del hipocampo) y de la corteza entorrinal. La porción posteromedial recibe las anteriores proyecciones, pero en menor cantidad. La región anterolateral recibe axones, principalmente de la corteza perirrinal, al igual que la región posterolateral. La corteza prefrontal proyecta a la parte anterior de este núcleo.

La amígdala cerebral también tiene proyecciones importantes a este núcleo. Su parte basolateral hace conexión con el accumbens anteromedial; la parte anterolateral del accumbens recibe axones de la porción central de la amígdala. La región ventral dopaminérgica del tegmento proyecta principalmente al accumbens medial. Las zonas vecinas a este núcleo dopaminérgico, de sus mismas características, proyectan al accumbens lateral. A la parte anterior del núcleo accumbens también llegan proyecciones del tálamo y, aunque en menor proporción, existen proyecciones desde el hipotálamo y el pálido ventral. La parte medial del núcleo accumbens (especialmente su parte anterior) recibe una variedad de proyecciones desde diversos núcleos, pero quizás la mayor proporción proviene del hipocampo, especialmente de sus células en las zonas $\mathrm{CA}_{1}, \mathrm{CA}_{2}$ y $\mathrm{CA}_{3}$. El subiculum también envía proyecciones importantes a esta área.

\section{Eferencias}

Las eferencias más reportadas de este núcleo son las que hacen sinapsis en regiones diencefálicas, corticales y del tallo cerebral (1113). Existen evidencias de proyecciones al hipotálamo, al cíngulo, al globo pálido, al tálamo, a la sustancia negra y a otras áreas de menor interés. Otras proyecciones consideradas de gran importancia en las funciones motoras, de conducta y de refuerzo para sustancias de abuso como la cocaína, las anfetaminas y otros estimulantes, son las que envía el núcleo accumbens a la llamada región motora mesencefálica. En esta región, se hace referencia especialmente a los núcleos pedunculopontinos del tegmento, los cuales tienen no sólo una función de regulación motora sino también una participación en el condicionamiento de la conducta y en las expresiones de condicionamiento a sicofármacos y a sustancias de abuso.

Las proyecciones que van al pallidum ventral son gabaérgicas; sin embargo, utilizando técnicas de microinfusión, se ha observado que la utilización de neurotransmisores excitadores o sus análogos en el núcleo accumbens, despierta una actividad locomotriz y de conducta de tipo excitadora. Estas observaciones sugieren, en conjunto, que existirían proyecciones tanto excitadoras como inhibidoras del núcleo accumbens a estructuras mesencefálicas responsables de la conducta, el refuerzo y de la actividad motriz.

\section{Hipocampo}

Es una estructura plegada, bien delineada, con algunas porciones de corteza, que tiene la forma de un caballito de mar (de donde toma su nombre) y que extiende su principal porción a lo largo del piso del cuerno temporal del ventrículo lateral. Se le puede considerar un giro de la alocorteza enrollada hacia dentro que cubre la fisura del hipocampo. A esta formación también se le conoce con el nombre de asta de Ammon y comprende varias partes. Precisamente por su diversidad celular y funcional, se le conoce como la formación del hipocampo de la cual hacen parte el subiculum, el giro dentado y el hipocampo propiamente dicho.

Esta estructura comenzó a ser estudiada desde los mismos inicios del presente siglo por Ramón y Cajal y por Lorente de Nó, cuyos escritos aún son clásicos. El hipocampo consta de tres regiones o capas corticales que se encuentran interrelacionadas recíprocamente y conectadas con otras estructuras límbicas, corticales y subcorticales. Las células más abundantes son las de tipo piramidal que se constituyen básicamente en las conexiones de proyección. Fue dividido por Lorente de Nó, en 1934, en región $\mathrm{CA}_{1}, \mathrm{CA}_{2}, \mathrm{CA}_{3}$ y $\mathrm{CA}_{4}$ (ahora llamada hilus dentado). La denominación $\mathrm{CA}$ corresponde a cornu ammonis $(14,15)$.

Esta estructura del hipocampo utiliza neurotransmisores inhibidores y excitadores para sus 
funciones. Es abundante la participación en sus funciones de receptores y neurotransmisores del tipo GABA, glutamato y aspartato. EI GABA -con mucha participación en circuitos locales- es el neurotransmisor inhibidor más abundante. El glutamato y el aspartato se utilizan mucho en las proyecciones largas que van al núcleo accumbens.

Con relación a las funciones atribuidas al hipocampo, algunos autores sostienen que esta formación no es el centro de una sola función, sino que es un tipo de relevo de muchas proyecciones a otras áreas del cerebro y, por tanto, involucrado en variadas actividades.

Al hipocampo se le ha involucrado en funciones moduladoras de conducta, emociones y procesos sicológicos. Además, tendría importante función en regular procesos o eventos motores, funciones endocrinas de comando hipotalámico, memoria (especialmente la reciente) y grados de vigilia. También es importante su actividad autónoma, a manera de marcapaso cerebral, la cual daría inicio a actividades excitadoras espontáneas, especialmente, las relacionadas con la disminución del umbral convulsivo (16).

Además, es de importancia considerar que las proyecciones glutamatérgicas y aspartatérgicas que llegan al núcleo accumbens desde el hipocampo, producen una liberación importante de dopamina en dicho núcleo. Esta acción hipocampo-accumbens induce el incremento de actividad de conducta y motriz en animales de laboratorio.

Este evento podría tener mucha relevancia en la acción de sicoestimulantes teniendo en cuenta que el núcleo accumbens se proyecta, a su vez, a núcleos mesencefálicos motores y de conducta.

\section{Conexiones del hipocampo}

\section{Aferencias}

Las fibras aferentes derivan de varias fuentes $(14,16)$. Estructuras muy importantes con relación a sus proyecciones a esta formación neuronal son la corteza entorrinal y perirrinal y el núcleo accumbens. Los núcleos u organizaciones del área septal envían proyecciones al hipocampo; también lo hacen áreas de la corteza prefrontal. Otros sitios que proyectan a esta estructura son el giro del cíngulo, el núcleo talámico anterior, cuyas proyecciones llegan, especialmente, a zonas subiculares del hipocampo. Las áreas visuales, auditivas, somatosensoriales y gustativas de la neocorteza llegan al hipocampo a través de contactos o sinapsis con el área entorrinal. De tal manera que el hipocampo recibe un rico y complejo aporte de aferencias relacionadas probablemente con funciones sensoriales, de memoria y de expresiones del sistema límbico.

No se deben desconocer los circuitos locales del hipocampo. Existe un sistema excitador unidireccional que se origina en los núcleos glutamatérgicos/aspartatérgicos que al entrar por la vía perforante desde las zonas olfatorias, estimulan las células granulares del giro dentado y éstas, mediante fibras musgosas, a las células $\mathrm{CA}_{3}$ piramidales, las cuales, a su vez, estimulan las piramidales de la zona $C A_{1}$, que envían proyecciones excitadoras a varios núcleos (como el núcleo accumbens). Cuando se estimulan las células piramidales de la zona $\mathrm{CA}_{3}$, ellas, con sus colaterales axónicas, estimulan otras neuronas piramidales homólogas; este es un circuito excitador local.

Con relación a los circuitos locales inhibidores, se conoce la gran participación del GABA con sistemas de retroalimentación negativa. También existe inhibición por vías directas aunque locales. El primer circuito local inhibidor cumple su función, principalmente, a través de las proyecciones de las interneuronas inhibidoras en forma de cesta. Este utilizaría receptores $\mathrm{GABA}_{\mathrm{A}}$ en células piramidales $\mathrm{CA}_{1}$ y $\mathrm{CA}_{3}$ y produciría una inhibición temprana. El segundo sistema inhibidor utilizaría interneuronas unidireccionales gabaérgicas que proyectarían axones que harían sinapsis presinápticas en los axones de las células $C_{1} A_{1}$ $\mathrm{CA}_{3} \mathrm{y}$, utilizando receptores $\mathrm{GABA}_{\mathrm{B}}$, producirían una inhibición presináptica tardía o lenta.

\section{Eferencias}

Las eferencias mayores del hipocampo son los axones de las células piramidales del área $\mathrm{CA}_{1} \mathrm{y}$ $\mathrm{CA}_{3}$. Estas se dirigen al núcleo accumbens, el subiculum, la corteza entorrinal, los núcleos 
septales, el bulbo olfatorio, la amígdala y el hipotálamo. Estas vías utilizan neurotransmisores excitadores como el glutamato y el aspartato para estimular sus respectivos receptores ubicados en las estructuras anteriormente mencionadas. Se pìnsa que esta conexión es muy importante en la función de estos núcleos.

\section{Neurofisiología y farmacología del sistema del placer y la drogodependencia}

Se han descrito las áreas cerebrales (llamadas sistema del placer, recompensa y abuso de drogas) donde se originaría el placer, donde se accionan los mecanismos que conllevan al refuerzo de conductas y la tendencia al abuso de ciertas drogas y fármacos. El sistema de refuerzo está constituido básicamente por estructuras del sistema límbico como el núcleo accumbens, el hipocampo, la amígdala, la sustancia negra, el pallidum ventral, los núcleos motores mesencefálicos y la corteza prefrontal (17).

Utilizando la técnica de microelectrodos implantados permanentemente en estas áreas de predominio dopaminérgico, a través de los cuales se administran microestímulos eléctricos después de que el animal ha efectuado un trabajo, se puede producir una conducta compulsiva progresiva por el trabajo, pues después de éste se recibirá un microestímulo eléctrico como recompensa. Este estímulo produce, supuestamente, una sensación agradable, placentera, en el animal de experimentación; por esta razón, se instaura un esquema de conducta, trabajo-estímulo-trabajo-estímulo, etc.

Se propone que para la ocurrencia de este fenómeno, se necesita de la participación de vías dopaminérgicas, especialmente los circuitos que van desde el mesencéfalo hasta el núcleo accumbens, el tubérculo olfatorio y las áreas límbicas relacionadas, como la corteza del cíngulo, la frontal, la entorrinal y la perirrinal, llamado sistema mesolímbico.

Se le ha dado un papel igualmente relevante en este fenómeno a los sistemas serotoninérgicos y noradrenérgicos. En humanos, mediante la técnica de tomografía de emisión de positrones, se ha demostrado la relevancia de estos sistemas en el fenómeno de abuso de la cocaína y de otras sustancias (18).

En este circuito se integran impulsos nacidos en el hipocampo y la amígdala, que activarían sucesivamente estructuras como el núcleo accumbens, el pallidum ventral, la corteza prefrontal y los núcleos motores mesencefálicos. Por esta activación se produciría inhibición de las vías inhibidoras y estimulación o liberación de las vías excitadoras. Esta acción resultaría en estados de excitación motora y de conducta.

La vía mesolímbica dopaminérgica modularía este circuito. La regulación dopaminérgica a nivel presináptico sobre las vías que llegan al núcleo accumbens desde el hipocampo y la amígdala, resultaría en disminución de la actividad inhibidora posiblemente mediada por GABA y liberación de las vías excitadoras glutamatérgicas y aspartatérgicas. Además, la dopamina, por sí misma, estimularía las vías excitadoras en el núcleo accumbens. Esto produciría incremento sicomotor por acción sobre el pallidum ventral y éste, a su vez, sobre los núcleos mesencefálicos motores y las áreas límbicas prefrontales (19).

En este circuito, es de gran relevancia la actividad del núcleo accumbens, en el cual hay un gran predominio de vías dopaminérgicas. Esta estructura es un puente importante de integración entre el sistema límbico y los sistemas motores. La manipulación electrofisiológica y farmacológica del núcleo accumbens ha puesto en evidencia la importancia que tiene en la regulación de los procesos motores y de conducta en roedores y primates (normales o con adicción).

Es ampliamente conocida la farmacología de las vías dopaminérgicas relacionadas con estas estructuras. La inyección bilateral de dopamina en el núcleo accumbens produce un marcado incremento de la actividad locomotriz, el cual puede ser bloqueado por fármacos neurolépticos, por la inyección local de muscimol, un agonista gabaérgico, o por elevación de las concentraciones de GABA a través de la inhibición de la enzima GABA transaminasa $(20,21)$.

El núcleo accumbens contiene, además, neurotransmisores excitadores como el ácido glutámico. 
Estudios in vitro han demostrado que el ácido glutámico libera dopamina en este núcleo. Adicionalmente, en estudios bioquímicos se ha observado que la infusión intra-accumbens de glutamato o agonistas de éste, induce la liberación de dopamina tanto in vitro como in vivo. Lesiones electrolíticas o neurotóxicas o la administración en el núcleo accumbens de bloqueadores de receptores dopaminérgicos $D_{2}$ (haloperidol) y de $\mathrm{D}_{1}(\mathrm{R}(+)-\mathrm{SCH} 23390)$, atenúan o eliminan los cambios de conducta inducidos por autoestimulación eléctrica o por administración de cocaína en ratas. Los antagonistas de los receptores de glutamato tipo NMDA (APV, ácido 2-amino-fosfono-valérico), tipo AMPA (DNQX: 6,7-dinitroqui-noxalina-2,3 diona) y tipo KAINATO (GAMS, sulfonato de $\gamma$-D-glutamilamino-metano) en el modelo de rata, bloquean los efectos estimulantes motores producidos por la cocaína. Por tanto, se cree que el glutamato podría jugar un papel importante en la regulación de la actividad locomotriz por su influencia en mecanismos dopaminérgicos y viceversa $(22,23)$.

Otra vía que podría tener importancia en el sistema de refuerzo en roedores y humanos son los neuropéptidos. La administración de $\beta$ endorfinas en el núcleo accumbens incrementa la liberación de dopamina y este efecto se acompaña de un aumento de la actividad locomotriz. Este efecto es atenuado por el antagonista de los receptores opioides delta $(\delta)$ $\mathrm{ICl}-174864$ que químicamente es el N, N-dialilTir-Aib-Aib-Fe-Leu (Aib, ácido a-aminobutírico) y por el antagonista de los receptores opioides mu ( $\mu$ ) CTOP, que es el D-Fe-Cis-Tir-DTrp-Orn-ThrPen-Thr- $\mathrm{NH}_{2}$. Se ha observado que la administración subcrónica de cocaína produce en el núcleo accumbens de ratas una disminución de $\beta$-endorfinas; estos resultados sugieren que la actividad de algunos neuropéptidos podría tener alguna importancia en el inicio de la cocainodependencia (24).

Este sistema de refuerzo puede ser afectado por otras sustancias como la nicotina, las anfetaminas, los barbitúricos, el alcohol, las benzodiazepinas, el LSD y la marihuana, las cuales, por sus propiedades para alterar el estado de ánimo, los sentimientos y la conducta, pueden crear dependencia en algunos individuos. Generalmente, en estos casos las personas requieren la administración de la droga para continuar con la sensación de bienestar físico y síquico que les produce.

La cocaína es un estimulante síquico que produce su efecto euforizante activando el mencionado sistema de refuerzo de conducta. Se ha demostrado que la activación del neurotransmisor dopamina en las vías llamadas mesolímbicas y mesocorticales es, en gran parte, responsable del refuerzo de conducta por estimulantes en roedores y en humanos. Los registros intracelulares in vivo en el núcleo accumbens, sugieren que la cocaína potencia la acción de la dopamina debido a la inhibición de su recaptación; por tanto, la cocaína inhibe el encendimiento (kindling) de las neuronas dopaminérgicas en este núcleo. El núcleo accumbens juega un papel muy importante como mecanismo disparador del comportamiento que conduce a la autoadministración de cocaína en roedores (21).

Las lesiones de las terminales dopaminérgicas en el núcleo accumbens por la 6-hidroxidopamina en el modelo de rata disminuyen la autoadministración de cocaína y afecta, en mucho menor grado, la autoadministración de heroína. Sin embargo, las lesiones de los sistemas neuronales eferentes del núcleo accumbens bloquean tanto la autoadministración de cocaína como la de heroína.

Después de la administración aguda de cocaína, se observa en el núcleo accumbens una disminución de la recaptación de dopamina, produciéndose como consecuencia un incremento en la actividad de este neurotransmisor lo cual explicaría, en parte, los efectos euforizantes y condicionantes que produce esta droga. Durante la administración crónica de cocaína, se observa una reducción en los niveles de dopamina y un aumento progresivo en la población de receptores alfa y beta adrenérgicos y dopaminérgicos pre y post-sinápticos. Estos son algunos de los efectos neurobioquímicos producidos por la cocaína en roedores (23). 
Lo anterior, tomado en conjunto, sugiere la importancia del núcleo accumbens y de sus proyecciones en los mecanismos moleculares responsables del refuerzo y dependencia de drogas como la cocaína, la heroína y otras sustancias de abuso en roedores y en humanos. La compulsión por la cocaína se explicaría por una mayor hipersensibilidad de los receptores dopaminérgicos presinápticos que de los postsinápticos, lo cual induciría un consumo de cocaína para mejorar temporalmente la disponibilidad de dopamina.

Todo lo anterior sustenta la idea de que los efectos agudos y crónicos de la cocaína son debidos a la alteración en la actividad de las vías dopaminérgicas y de sus receptores; también estarían involucradas otras vías monoaminérgicas y sus receptores. Se utilizan agonistas dopaminérgicos como la bromocriptina y sustancias que aumentan la disponibilidad de dopamina como la amantadina, la cual hace parte del tratamiento integral de estos pacientes. Otra alternativa en el tratamiento farmacológico del abuso de cocaína, ha sido la administración de un agonista parcial opioide como la buprenorfina.

Fármacos como los antidepresivos tricíclicos (especialmente, la desipramina y la imipramina) son capaces de mejorar significativamente el comportamiento compulsivo por la droga y el estado anhedónico en los cocainómanos.

También se reconoce el posible papel del sistema serotoninérgico en el estado anímico y de cocainodependencia. De acuerdo con esto, se han ensayado experimentalmente inhibidores de la recaptación de serotonina (como la fluoxetina) en modelos de cocainodependencia en ratas (25).

Este enfoque ha dado soporte a aproximaciones terapéuticas que tratan de corregir los trastornos mencionados. Sin embargo, no se obtiene una consistencia en los resultados con los pacientes, lo cual indicaría que otros sistemas estarían jugando un papel importante en las manifestaciones neurosicotóxicas causadas por la cocaína.

Se ha descrito el efecto de la cocaína en áreas del hipocampo y prefrontales en el ser humano, dando cuenta de la forma en que la ingestión de cocaína produce gran activación bioeléctrica en estas áreas, acompañada de un incremento sicomotriz, lo que puede deberse a una disminución de la actividad gabaérgica en estos núcleos (26).

Evidencias electrofisiológicas en roedores, indican que las neuronas del núcleo accumbens muestran una respuesta excitadora a la estimulación del hipocampo por medio de vías glutamatérgicas. Las respuestas locomotriz y de conducta tendrían un fondo neurobioquímico en la actividad de glutamato y aspartato, los cuales, al ser liberados en las sinapsis hipocampo-núcleo accumbens, liberan dopamina de las aferencias mesolímbicas dopaminérgicas en dicho núcleo. Esta actividad en el núcleo accumbens activaría los centros mesencefálicos que serían los responsables del funcionamiento de la conducta y motriz (22).

La cocaína incrementaría la intensidad de las respuestas en estos núcleos al cambiar drásticamente la actividad recíproca dopamina/ gaba y glutamato-aspartato. Esta idea está sustentada por nuestros hallazgos al administrar cocaína y medir los niveles de GABA, glutamato y aspartato en el núcleo accumbens y en el hipocampo de rata, utilizando técnicas enzimáticas $(27,28)$ (cuadro 1).

Tomada toda esta información en conjunto, podemos resumir que la acción y los efectos agudos y crónicos causados por drogas o fármacos de abuso que producen dependencia, tienen un substrato anatómico y neurobioquímico en áreas cerebrales (especialmente, el núcleo accumbens, el hipocampo, la corteza prefrontal, la amígdala y los centros motores mesencenfálicos), donde se producirian alteraciones, principalmente en neurotransmisores y receptores dopaminérgicos, glutamatérgicos, aspartatérgicos y gabaérgicos.

Vale la pena resaltar en esta actualización, la relevancia del papel que juegan los neurotransmisores aminoácidos en la fisiología del sistema de recompensa y en la drogodependencia, especialmente en el fenómeno descrito (20).

Muy recientemente se ha ligado la actividad del óxido nítrico en este circuito neuronal y con los 
Cuadro 1. Efectos de la cocaína sobre los niveles de GABA, glutamato y aspartato en el nucleo accumbens e hipocampo de rata (28).

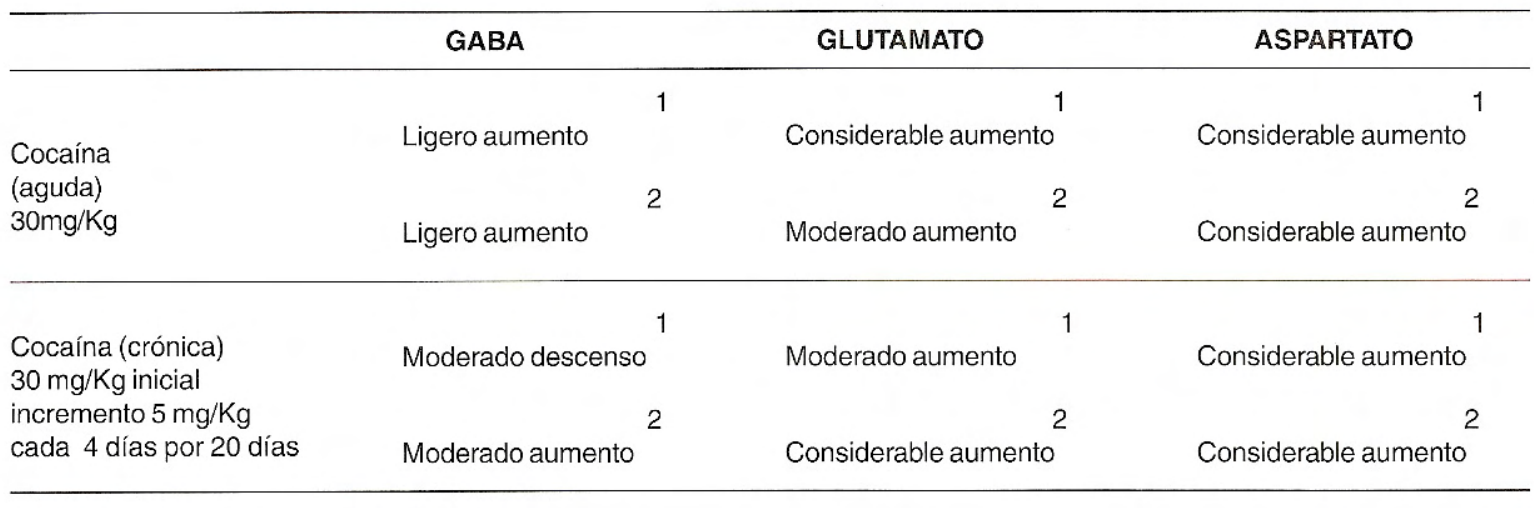

1= Núcleo accumbens $\quad 2=$ Hipocampo

mecanismos de la drogodependencia. Se postula que el incremento de la actividad sicomotriz por drogas de abuso se debe al incremento de los niveles de este neuromensajero; ello debido al estímulo de la actividad de la óxido-nítricosintetasa por cambios en los receptores NMDA post-sinápticos, debido a la acción del glutamato y del aspartato sobre ellos (4, 29-32).

\section{Agradecimientos}

Al señor Iván Carlos González por su asistencia en el manejo del texto y de las gráficas.

\section{Referencias}

1. Markou A, KostenTR, Koob GF. Neurobiological similarities in depression and dependence: a self-medication hypothesis. Neuropsychopharmacology 1998;18:135-74.

2. Enna SJ, Wood JH, Snyder SH. GABA in human cerebrospinal fluid: radio-receptor assay. J Neurochem 1977;28:1121-24.

3. Chang JY, Janak PH, Woodward DJ. Comparison of mesocorticolimbic neuronal responses during cocaine and heroin self-administration in freely moving rats. J Neurosci 1998;18: 3098- 115.

4. Bardo MT. Neuropharmacological mechanism of drug reward: beyond dopamine in the nucleus accumbens. Crit Neurobiol 1998;12:37-67.

5. Comings DE, Muhleman D, Gade R, Johnson P, Verde $\mathbf{R}$, Saucier $\mathbf{G}$ et al. Cannabinoid receptor gene (CNR1): association with i.v. drug use. Mol Psychiatry 1997;2:161-8.

6. Heimer L, Wilson RD. The subcortical projections of the allocortex: similarities in the neural associations of the hippocampus, the piriform cortex, and the neocortex. En: Santini M, editor. Perspectives in neurobiology. New York: Raven Press, 1975;177-93.
7. Yim CY, Mogenson GJ. Response of ventral pallidal neurons to amygdala stimulation and its modulation by dopamine projections to nucleus accumbens. J Neurophysiol 1983;50:148-61.

8. Tassin JP. Drogas, dependencia y dopamina. Mundo Científico 1998;189:68-73.

9. Dahlstrom A, Fuke K. Evidence for the existence of monoamine containing neurons in the central nervous system. I. Demostration of monoamines in the cell bodies of brainstem neurones. Acta Physiol Scand 1964;62:1-55.

10. Phillipson OT, Griffiths AC. The topographic order of inputs to nucleus accumbens in the rat. Neuroscience 1985;6:275-96.

11. Inglis WL, Dunbar JS, Winn P. Outflow from the nucleus accumbens to the pedunculopontine tegmental nucleus. A dissociation between locomotor activity and the acquisition of responding for conditioned reinforcements stimulated by d-amphetamine. Neuroscience 1994;62:51-64.

12. Jones DL, Mogenson GJ. Nucleus accumbens to globus pallidus GABA projection: electrophysiological and iontophoretic investigations. Brain Res 1980;188:93-105.

13. Donzanti BA, Uretsky NJ. Effects of excitatory amino acids on locomotor activity after bilateral microinjection into the rat nucleus accumbens; possible dependence on dopaminergic mechanisms. Neuropharmacology 1983;22:971-81.

14. Lorente De Nó R. Studies on the structure of the cerebral cortex. II. Continuation of the study of the Ammonic system. J Psychol Neurol 1934;46:113-77.

15. Knowles WD. Normal anatomy and neurophysiology of the hippocampal formation. J Clin Neurophysiol 1992;9:25263.

16. Rector DM, Poe GR, Harper RM. Imaging of hippocampal and neocortical neural activity following intravenous cocaine administration in freely behaving cats. Neuroscience 1993;54:633-41. 
17. Tzschentke TM, Schmidt WJ. Discrete quinolinic acid lesions of the rat prelimbic prefrontal cortex affect cocaineand MK-801-, but not morphin-and amphetamine-induced reward and psychomotor activation as measured with the place preference conditioning paradigm. Behav Brain Res 1998;97:115-27.

18. Volkow ND, Fowler JS, Wolf AP. Use of positron emission tomography to study cocaine in the human brain. En: Rapara RS, Makriyannis A, Kuhar MJ, editors. Emerging technologies and new directions in drug abuse research. Natl Inst Drug Abuse Res Monogr Ser. U.S. Washington D.C.: Government Printing Office; 1991;112:168-79.

19. Peoples LL, Gee F, Bibi R, West MO. Phasic firing time locked to cocaine self-infusion and locomotion: dissociable firing patterns of single nucleus accumbens neurons in the rat. J Neurosci 1998;18:7588-98.

20. Kushner SA, Dewey SL, Kornetsky C. Gamma-vinyl GABA attenuates cocaine-induced lowering of brain stimulation reward thresholds. Psychopharmacology 1997;133:383-8.

21. Morgan AE, Dewey SL. Effects of pharmacologic increases in brain GABA levels on cocaine-induced changes in extracellular dopamine. Synapse 1998;28:60-5.

22. Karler R, Carlder LD. Excitatory amino acids and the action of cocaine. Brain Res 1992;582:143-6.

23. Karler R, Calder LD, Thai LH, Bendingfield JB. The dopaminergic, glutaergic, gabaergic bases for the action of amphetamine and cocaine. Brain Res 1995;671:100-4.

24. Spanagel R, Herz A, Bals-Kubik R, Shippenbert TS. Beta-endorphin-induced locomotor stimulation and rein- forcement are associated with an increase in dopamine release in the nucleus accumbens. Psychopharmacology 1991;104:51-6.

25. Carrol ME, Lac ST, Asencio M, Kragh R. Fluoxetine reduces intravenous cocaine self-administration in rats. Pharmacol Biochem Behav 1990;35:237-44.

26. Ye JH, Liu PL, Wu WH, McArdle Ju. Cocaine depresses GABA current of hippocampal neurons. Brain Res 1997;770:169-75.

27. Graham LT, Aprison MH. Distribution of some enzymes associated with metabolism of glutamate, aspartate, gaba and glutamine in cat spinal cord. J Neurochem 1969;16:55966 .

28. Ulloque RA. Efectos de la cocaína sobre los niveles de gaba, glutamato y aspartato en el núcleo accumbens e hipocampo de rata. Febrero 1999 ; Tesis Doctoral Universidad de Sevilla (España).

29. Kim JH, Vezina P. Metabotropic glutamate receptors in the rat nucleus accumbens contribute to amphetamine-induced locomotion. J Pharmacol ExpTher 1998;284:317-22.

30. Hölscher $\mathbf{C H}$. Nitric oxide, the enigmatic neuronal messenger: its role in synaptic plasticity. TINS 1997;20:298303.

31. ItzhakY, Gandia C, Huang PL, Ali SF. Resistance of neuronal nitric oxide synthetase-deficient mice to methamphetamine-induced dopaminergic neurotoxicity. J Pharmacol ExpTher 1998;284:1040-7.

32. Filip M, Przegalinski E. The role of nitric oxide (NO) pathway in the discriminative stimuli of amphetamine and cocaine. Pharmacol Biochem Behav 1998;59:703-8. 